\title{
Medical technology Innovation for a sustainable impact in Low- and Middle-Income countries: a holistic approach.
}

Solomzi Makohliso a, Bertrand Klaiber ${ }^{\mathrm{b}}$, Romain Sahli ${ }^{\mathrm{b}}$, Jean Roger Moulion Tapouh ${ }^{\mathrm{c}}$, Samuel Nko'o Amvene $^{\mathrm{c}}$, Beat Stoll ${ }^{\mathrm{d}}$, Klaus Schönenberger ${ }^{\mathrm{a}, \mathrm{e}}$

\begin{abstract}
Technologies that have been designed for use in high-income countries often fail to deliver their full potential when transposed to Low and Middle-Income Contexts (LMICs). The health sector is a case in point, as medical devices, whether donated or purchased, are generally short lived in those contexts. The mismatch between needs and available solutions originates from the inadequacy of both the technology and the business models. Essential medical technologies such as oxygen concentrators, neonatal incubators, anesthesia machines or diagnostic X-ray systems are classic examples. The case of diagnostic Xray imaging is particularly striking: 125 years after its invention, up to two thirds of the world population still does not have access to radiology services, according to the World Health Organisation. This is despite the fact that X-ray radiology is one of the cornerstone of healthcare and a crucial instrument for diagnosing a variety of health issues ranging from trauma to tuberculosis and other lung diseases.

We are presenting an integrated methodological approach, to develop innovative solutions adapted to the context of LMICs. The approach relies on three crucial pillars: cooperation, interdisciplinarity and entrepreneurship with a long-term sustainability perspective. We propose a set of four complementary tools that increase the chances of successfully developing and deploying the technologies at scale. The tools, while very practical, allow striking a balance between economic viability, environmental and social impact. We illustrate the use of these tools with the case of diagnostic X-ray imaging.

We propose that using the approach and tools presented here could allow to rethink other complex technologies that have the potential to address social challenges, in the perspective of making them suitable for LMICs. We also believe that this approach to developing solutions addressing the needs of poorer communities, may lead to better products in industrialized contexts as well.
\end{abstract}

\section{INTRODUCTION}

The seventeen Sustainable Development Goals (SDGs) of the 2030 UN Agenda call for action by all countries to unite and promote social prosperity while protecting the planet. Technology impacts all the SDGs, as it serves a critical role in fulfilling basic human needs, such as food, health, shelter or education, as well as underpinning economic prosperity. For the purpose of this discussion, we define essential technologies as those technologies that can expedite the achievement of the SDGs ${ }^{*}$. While most of these essential

a Ecole Polytechnique Fédérale de Lausanne (EPFL), EssentialTech Centre, Switzerland.

${ }^{b}$ Ecole Polytechnique Fédérale de Lausanne (EPFL), Program EssentialTech, Switzerland. Now Pristem SA, Switzerland.

c Faculty of Medicine and Biomedical Sciences, and Department of Medical Imaging and Radiation Therapy, University of Yaoundé I, Cameroon.

d EssentialMed Foundation, Switzerland, and University of Geneva, Global Health Institute, Switzerland.

e Corresponding Author.

* According to our analysis, they are specifically to be found in the fields of information and communication, energy, agriculture, nutrition, medical devices, pharmaceuticals, water \& sanitation, and transportation technologies. 
technologies are widely available in industrialized countries, many of them have not been successfully deployed on a large scale in Low and Middle-Income Countries (LMICs). Of all the essential technologies, the medical devices sector lags considerably. According to the World Health Organization (WHO): "Four fifths of global medical device sales revenue comes from sales in the Americas and Europe. Ten countries account for nearly $80 \%$ of world sales revenue, with the United States at the top of the list (41\%), followed by Japan (10\%), Germany ( $8 \%)$, and France $(4 \%)^{\prime \prime}$. The imbalance in access between these industrialized countries and LMICs has been attributed primarily to high costs, technological complexity and also to a significant disregard of the unique constraints inherent to LMICs when developing these products. This disregard can lead to technology mismatches that engender high levels of premature device failure in LMIC contexts.

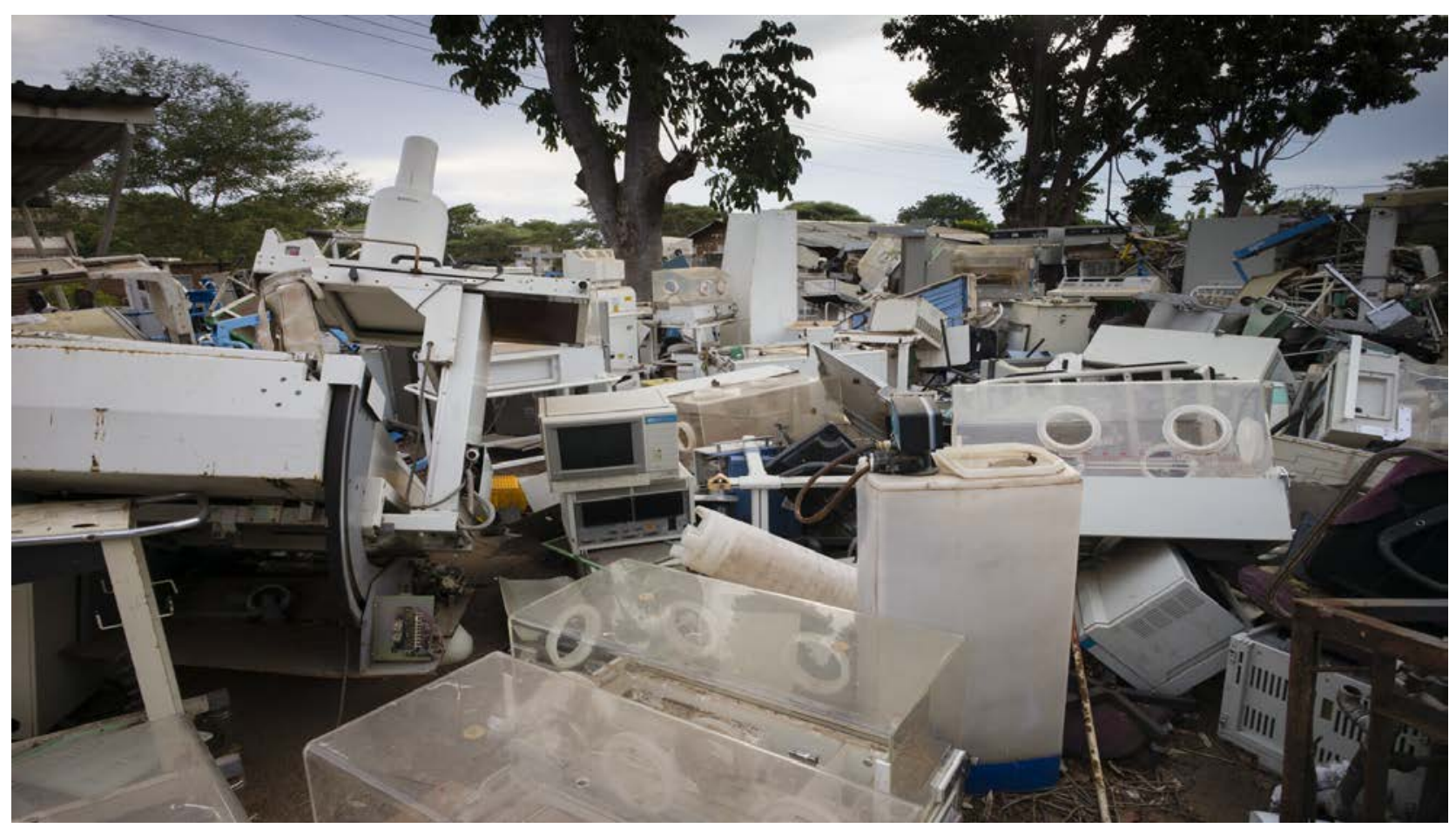

Figure 1 Classic view of abandoned medical devices in the backyard of a hospital in Malawi. For the most part, these devices were acquired via donations. Note the presence of several neonatal incubators, in spite of the fact that they are sorely needed locally. (CGradian Health, with permission

Figure 1 illustrates this mismatch. It shows medical devices abandoned in the backyard of a hospital in Africa. Sadly, such scenes are ubiquitous in many LMICs. Currently, most medical equipment used in lowresource settings is imported or donated from industrialised countries. Unfortunately, only $10-30 \%$ of donated medical equipment becomes operational at the destination countries, according to WHO estimates ${ }^{1}$. This ultimately results in high but potentially preventable mortality rates, as illustrated by the following examples.

Oxygen Concentrators: In paediatrics, hypoxaemia is the major fatal complication of pneumonia, the leading cause of death from infectious diseases in children under the age of five years worldwide, accounting for more childhood deaths than HIV/AIDS, malaria, and tuberculosis combined. Oxygen concentrators provide a sustainable and cost-effective source of medical oxygen (when compared with oxygen cylinders) for the treatment of hypoxaemic patients by providing concentrated oxygen $(93 \% \pm 3 \%)$ to stabilise their arterial oxygen saturation levels. In high-income countries where suitable personnel and resources are available for maintenance, oxygen concentrators have a life expectancy of 7 years or more, depending on usage and frequency of maintenance. In contrast, concentrators used in LMICs have a much shorter lifetime due to 
harsh operating environments (high heat, humidity and dust levels), poor quality power and a lack of basic maintenance tasks such as filter changes ${ }^{2}{ }^{3}$. A recent study in Lao People's Democratic Republic reported $37 \%$ of recently procured concentrators experienced a fault requiring maintenance within a year of their installation ${ }^{4}$ and a study in Nigeria ${ }^{5}$ found that of 57 'working' concentrators tested, only 2 were fit for use (i.e. producing $>85 \%$ oxygen and electrically compatible).

Neonatal Incubators: An estimated 15 million babies are born too early every year, where almost 1 million children die each year due to complications of preterm birth. In low-income settings, half of the babies born at or below 32 weeks ( 2 months early) die due to a lack of feasible, cost-effective care, whereas in highincome countries, almost all of these babies survive (source WHO fact sheets ${ }^{6}$ ).

It has been reported that prevention and management of hypothermia could avoid up to $40 \%$ of neonatal deaths in developing countries ${ }^{7}$. However, due to lack of functional neonatal incubators, health care facilities in developing countries are often unable to properly manage hypothermia in newborns, especially in cases where skin-to-skin warming with a parent (aka 'kangaroo care') are not applicable or indicated (e.g. when the child is sick and needs medical attention). Although several initiatives attempted to tackle this need for infant incubators and other neonatal care devices ${ }^{8}{ }^{9}$ as well as countless local designs in the frame of student projects, none has been successful in reaching any scale.

Anaesthesia Machines: The Lancet Commission on Global Surgery estimates some 5 Billion people have inadequate access to safe surgery due to failure modes in the complex perioperative ecosystem ${ }^{10}$. In the operating theatre a single malfunctioning device may preclude surgery, requiring referral to distant hospitals or, in emergency cases, cause significant morbidity and mortality. A survey of hospitals in Malawi found that $50 \%$ did not have a functioning anaesthesia machine, including donated equipment with no product training from the donor or service support from the original manufacturer ${ }^{11}$. Furthermore, most anaesthesia machines require compressed oxygen and mains power, which, in many underfunded facilities, is frequently unavailable. One non-profit company has addressed this through a combination of technical design ${ }^{12}$, expanded simulation-based product training conducted by local physician anaesthesiologists recreating real-life conditions, and investment in local bioengineering capacity to ensure equipment can be maintained in operating condition and urgent service provided in a timely manner. This model, which has hybrid for-profit and not-for-profit characteristics, has been deployed successfully in over 700 LMIC hospitals, and been used to train hundreds of clinical providers. However, it relies on significant philanthropic funding and has yet to provide a model as a scalable, self-sustaining and entirely marketbased solution.

Diagnostic X-ray imaging: According to the WHO, between two-thirds and three-fourths of the world's population do not have proper access to radiology services ${ }^{13}$. X-ray radiology is a crucial instrument in healthcare for diagnosing a variety of health cases. It is essential for the diagnosis and management of pulmonary diseases, such as tuberculosis and pneumonia, which still represent a high burden in LMICs. For trauma, it is vital for cases such a road traffic accidents, of which $90 \%$ occur in $\mathrm{LMICs}^{14}$, precisely where this device is lacking.

The poor access to essential medical devices above arises from failures to adapt the conception and deployment strategies of these devices to the contexts of LMICs $^{15}$. However, innovations that are adapted to such contexts are sorely needed, in order to enable access to the essential benefits they confer. On the other hand, they could also represent new commercial opportunities and markets for medical equipment manufacturers.

This article is intended for researchers, engineers, as well as budding entrepreneurs primarily in academic and research institutions, who are interested in accelerating universal access to essential technologies, notably medical devices, in developing and emerging countries. 
We propose and discuss a holistic methodology, which advocates going beyond the traditional practices employed in academic research settings for medical device innovation. This multi-dimensional approach has also been inspired by established industrial, entrepreneurial and social science perspectives, where one of the main goals is to reduce risks associated with the innovation and facilitate its subsequent commercial viability and deployment for large-scale social impact.

We illustrate this proposed approach via a technically complex example, diagnostic X-ray imaging, in order to examine how it could be rendered more accessible globally in a sustainable manner by completely rethinking its conception. We expect that it should be possible to extend the applicability of this methodology to other medical devices, including the examples mentioned earlier.

\section{Approach}

Since the goal is to simultaneously accomplish commercial viability and large-scale health and social impact, it is important to develop a holistic understanding of the context where the product will be used, as opposed to primarily or exclusively concentrating on the technological aspects. The main challenge is to develop sustainable solutions -from a technological, ecological and an economic perspective - that are affordable, adapted to local needs and constraints, as well as scalable. Due consideration has to be given as to how the innovative technology will be deployed and sustained throughout the product life cycle, including its proper decommissioning. It is necessary to develop strategies that achieve both social impact and commercial success, in a mutually reinforcing manner: numerous models exist that allow a blend of varying degrees of social impact and financial return.

As Prahalad elegantly argued ${ }^{16}$, LMICs represent major untapped opportunities and should not be considered as mere philanthropic beneficiaries. While conventional marketing approaches remain relevant, it also necessary to give due consideration to certain critical differences between High-Income Countries (HICS) and LMICs. The importance and the need to rethink approaches to engineering devices that can succeed in LMICs has been discussed in the past ${ }^{17}$. Here we aim to complement these efforts by introducing a more holistic approach, including practical tools that are based on widely used entrepreneurship, social impact and sustainability principles. In the ensuing sections, we introduce four strategic tools to help structure the technology innovation process destined for LMICs, which we have framed in the following questions:

1. What: What is the current social situation (unmet need) and how do we draft a roadmap in order to achieve relevant social impact? This question is ideally answered using the Impact Canvas tool.

2. Where: Contextual factors may affect the performance and access to the essential technology under consideration. For this, we proposed a Context Analysis canvas.

3. Who: Who are the key stakeholders (global and local) that we need to involve in our project to assure successful deployment, uptake and long-term effective use of the technology? Here we propose a Value-Chain canvas.

4. How: How can we make the impact sustainable and economically viable in the long term? How can our venture be self- sustained while achieving the social goal? This requires the use of the Sustainable Business Model canvas.

These questions represent four different lenses through which we can look at the problem our technology innovation aims to solve and how to best structure the solution. We briefly present these tools in the following paragraphs, using the example of the medical X-ray technology, and Cameroon as a pilot country, to illustrate how they could be applied.

All the tools presented here are intended to be used in workshops where participants are knowledgeable in the specific domains treated. It is particularly important for the stakeholders from LMICs to participate in such discussions. In the case of our X-ray example, this would include the participation of radiologists, radiographers, medical doctors, engineers, patients, etc. They provide invaluable inputs without which the 
tools may not deliver their full potential. It is important to point out that the exercise of using the tools will in itself help to reveal and identify the stakeholders.

The tools are intended to be used iteratively throughout the technology development process, as the information and strategies are most certainly likely to evolve during the project. They also need to be used in parallel as they are all interconnected: outputs from one of them will be inputs for another one. Finally, in general such brainstorming sessions work best when they are fun and informal, e.g. using a large printed poster version of the tool with posted notes, refreshments and a good deal of humor.

WHAT? Roadmap to impact In order to ensure that an innovative venture provides positive social benefits, it is necessary to define the desired long-term goals at the very onset of our activities. The pathway to the desired impact involves clearly understanding the current situation and then determining all the steps and resources required to generate the effects that ultimately lead to the impact.

It is advisable to start by accurately identifying the unmet need to be addressed, we call this the situation. The next step is then to determine the inputs (resources, skills, people, infrastructure, etc.) required, as well as gaining a sound understanding of the context. Activities are a set of actions or tasks that the organization needs to perform with the inputs, in order to help realize and support the impact objectives. As a consequence, outputs will be generated, which can be defined as the tangible products and services that result from the activities undertaken. These outputs will then cause changes or effects on individuals or the environment, which are the outcomes that you expect to result from the delivery of products and services. Finally, the outcomes can lead to the much desired impact, which is defined as the effects and/or changes in society or the environment that follow from the outcomes that have been achieved.

One of the most commonly used methods to describe and map out the pathway to impact is the "Theory of Change" ${ }^{18}$. It generates what we term, for the purposes of this article, an Impact Plan, which enables a clear identification and distinction between expected outputs, outcomes, and impact. It also enables the identification of critical steps and required resources, as well as the anticipation of alternative plans if the present plan is inadequate or not suitable.

As an illustration, in the case of the X-ray, a simplified example of the impact plan is shown in Table 1 below.

\begin{tabular}{|c|c|c|c|c|c|}
\hline Situation: & Inputs: & Activities: & Outputs: & Outcomes: & Impact: \\
\hline $\begin{array}{l}\text { Low access to } \\
\text { radiology resulting } \\
\text { in high morbidity } \\
\text { /mortality. }\end{array}$ & $\begin{array}{l}\text { Context } \\
\text { Information } \\
\text { Medico- } \\
\text { technical } \\
\text { expertise, } \\
\text { Purchasing } \\
\text { power. } \\
\text { Infrastructure } \\
\text { challenges. }\end{array}$ & $\begin{array}{l}\text { R\&D. } \\
\text { Development of } \\
\text { training modules } \\
\text { for machine } \\
\text { operation \& } \\
\text { maintenance. } \\
\text { Industrialization. } \\
\text { Development of } \\
\text { appropriate } \\
\text { marketing and } \\
\text { servicing } \\
\text { strategies. }\end{array}$ & $\begin{array}{l}\text { Robust, affordable X-ray } \\
\text { machines, Training modules } \\
\text { available. Industrial strategy to } \\
\text { market and support } \\
\text { technology, digital services } \\
\text { (cloud-based image storage and } \\
\text { remote diagnosis). } \\
\text { Reduction in operating costs } \\
\text { and clear economic model for } \\
\text { acquisition }\end{array}$ & $\begin{array}{l}\text { Increased access } \\
\text { \& precise } \\
\text { diagnosis; skilled } \\
\text { personnel; } \\
\text { proper, safe and } \\
\text { sustained } \\
\text { operation. }\end{array}$ & $\begin{array}{l}\text { Reduction in } \\
\text { morbidity \& } \\
\text { mortality due to } \\
\text { trauma, tuberculosis } \\
\text { and other medical } \\
\text { conditions requiring } \\
\text { medical imaging. }\end{array}$ \\
\hline
\end{tabular}

Table 1: impact canvas tool, filled-in with specific data for the X-ray example

The tool clearly indicates that the mere design and subsequent production of a cost-effective robust digital imaging system (which is an output), does not automatically imply a long-term reduction in the morbidity and mortality relating to orthopedic traumas and pulmonary diseases, which is the impact which would be hoped for. There needs to be some intermediary outcomes, meaning that the technology needs to be accessible in hospitals, along with suitably trained staff, as well as continued support in terms of maintenance, repairs and quality of care.

The Impact Plan can help uncover alternative paths and provide important insights about the quickest and most efficient strategy to reach impact and scale. For instance, in one can envisage creating a start-up 
company or, alternatively, it could be more relevant to transfer or license out the innovation to an existing company with compatible interests.

Finally, the Impact Plan also involves the selection of methods to measure and track progress towards outputs, outcomes and impact via the use of carefully chosen indicators/metrics, which can sometimes be challenging, notably for impact.

WHERE? The Context Analysis tool. The context in which technologies are placed and used in LMICs presents considerable challenges $^{18}$. We noted that these challenges can be broadly and rationally grouped into five categories (Fig. 2). We present each of these categories and illustrate the learnings which can be drawn from the use of the tool in the example of diagnostic X-ray imaging. i) Lack of Financial resources. LMICs are, by definition, countries where financial resources are scarce (according to the World Bank, Cameroon's health expenditure per capita for 2016 in international dollars was 169 at purchasing power parity, compared to 4'178 for the UK and 9'870 for the United States). This affects not only their ability to purchase the technology in the first place but also to pay for services and consumables related to the technology. When financial resources are scarce and operational budgets

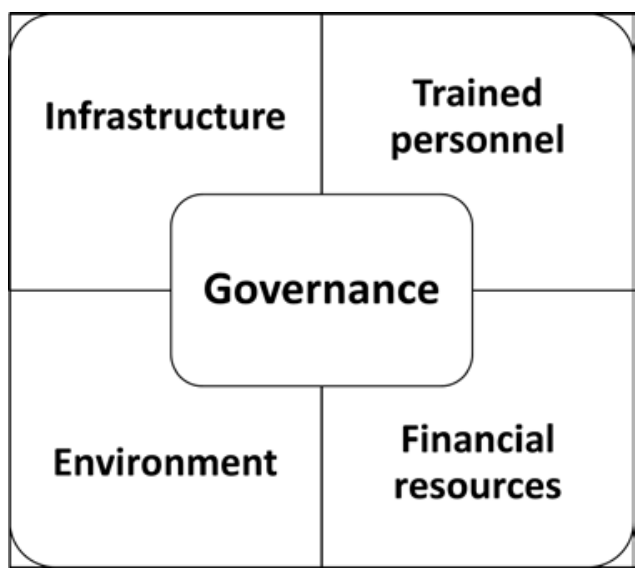

Figure 2 Context analysis canvas unpredictable, we propose that it makes better economic sense to consider the Total Cost of Ownership (TCO) of the device, rather than the initial purchase cost alone. This is because most of these devices typically have recurring costs for them to continue functioning (e.g. maintenance contracts, consumables), and thus periodic budgetary deficits can be a crippling factor for sustainable social impact. TCO calculation involves adding up all the costs incurred by the owner of a product, including the initial purchase price, taxes, transport, commissioning, training, operational costs and consumables, maintenance, repairs and disposal, during the complete life cycle of the product. Unfortunately, many buyers of medical equipment in LMICs especially still focus on acquisition costs and fail to evaluate product life cycle expenses, including consumables and maintenance. If they did, they would either plan accordingly for those costs (if they have the means) or choose an entirely different product. Currently, digital X-ray solutions have struggled to take hold in LMICs due to their high TCO and/or have not shown to work sustainably due to their fragility with respect to the context (e.g. Computed Radiography). The most prevalent X-ray technology in LMICs is still the analogue system that requires film plates to obtain the image. At first glance, this looks to be an affordable option, if you only focus on the initial purchase price. However, a closer look reveals non-negligible recurring costs from consumables (X-ray film plates and chemicals) and maintenance costs, where the annual maintenance contracts are typically $8 \%$ of the purchase price or higher in LMICs. We have carried out a simplified TCO calculation for a diagnostic X-ray that considers a lifecycle of 10 years (Table 2). Taking into account the purchase price, consumables and maintenance costs the calculation yields a TCO of US\$544'000, of which $84 \%$ are consumables. More extensive models would typically include additional dimensions such as training costs, energy consumption, floor space etc. Furthermore, the problem of weak supply chains in LMICs results in shortages of key consumables, such as film and developing supplies, which effectively blocks such film-based machines from providing access to the essential service. Based on this analysis, one can see that one of the key strategies to improve access $\mathrm{X}$-ray radiology is to propose a machine that eliminates or reduces consumables to a minimum, such as a digital $X$-ray machine. However, digital X-ray machines do exist, but come at premium acquisition costs well beyond the scope of many LMIC budgets. 


\begin{tabular}{|c|c|c|}
\hline & USD & Comments \\
\hline Purchase & $35^{\prime} 000$ & Analog machine \\
\hline Consumables 5d/week & $455^{\prime} 000$ & $\$ 3.5 /$ film incl. chemicals, 50 films /day, 10 years \\
\hline Uninterruptible power supply & $10^{\prime} 000$ & $\begin{array}{l}\text { one-time purchase } \\
\text { (maintenance costs are neglected here for simplicity) }\end{array}$ \\
\hline Maintenance $10 \mathrm{yr}$ & $28^{\prime} 000$ & Typically $8 \%$ of initial purchase price/year \\
\hline Repairs & $16^{\prime} 000$ & 1 tube, 1 cassette, Labour \\
\hline $\begin{array}{l}\text { Total Cost of Ownership (TCO) } \\
\text { Estimate for } 10 \text { Years }\end{array}$ & $544^{\prime} 000$ & $\begin{array}{l}\text { Not included: radiation protection equipment, automatic film } \\
\text { processor, staff training, electricity and water supply, } \\
\text { radiographic room infrastructure etc. }\end{array}$ \\
\hline
\end{tabular}

Table 2: Total cost estimate for an analogue X-ray system

Therefore, the challenge is to optimize costs for the complete lifecycle, not just the initial purchase price. If the device can be designed intelligently such that it is robust and requires very little maintenance over its lifetime and no consumables, then if a TCO approach is adopted it would likely represent a more affordable and sustainable approach towards improving access to X-ray radiology in many LMICs, even if the initial purchase price is higher than the film-based machines. Thus, the technology's lifetime is a key parameter and it seems reasonable to aim for a lifetime of 10 years for such a piece of capital equipment.

ii) Lack of Quality Infrastructure. Lack of quality infrastructure in key areas such as electricity supply, roads, water supply and buildings poses a major challenge. Poor electricity ranks as the highest infrastructural challenge for medical devices in LMICs: frequent power outages reduce equipment availability, while electrical perturbations (spikes and sags) are highly deleterious and account for about $30 \%$ of medical equipment damage ${ }^{19} 20$.

In order to confront these challenges, it is necessary to rethink the design of power supply circuits, which are the main unit in direct contact with electricity grid. In the case of the X-ray machine, it should be protected against grid instabilities (e.g. electrical surges). Given the poor quality of electrical installations in small hospitals in LMICs, it would be advisable to design the power supply to accept simple AC $(110 / 220)$ power sources rather than three-phase supply that are more difficult to install. The incoming power from the grid should go through a stage or device that is immune from power surges and can accept and store away all the power delivered (e.g. super-capacitors). The power supply should also include a lowmaintenance battery with a long lifetime that is capable of assuring continuous function for at least one hour in the event of a power failure; this would eliminate the need for expensive and maintenance-intensive external uninterruptible power supplies (UPS). One hour would generally be sufficient time to allow the hospital personnel to bring up a back-up generator, in the event of an extended power outage. Furthermore, it may require $30-50 \mathrm{~kW}$ for a split of a second to take an X-ray radiograph, which is often equivalent or even above to the total power available to a small hospital in LMICs. Thus, it would better to design the machine such that its overall consumption is considerably less than that, e.g. $1 \mathrm{~kW}$ or less. It could achieve this by avoiding to draw power from the grid directly, but rather from a storage device within the power supply (e.g. via super-capacitors).

iii) Harsh Environment. In many LMICs, the environment within the hospitals is typically hot, humid and dusty, which can be highly damaging to medical technology products and will reduce their longevity. The climate in some LMICs is characterised by temperatures in excess of $40^{\circ} \mathrm{C}$ and close to $100 \%$ humidity, which fall outside the product specifications of most medical devices.

For the X-ray, a case in point is the positioning mechanism of the source-detector gantry with respect to the patient. It must not only be simple and user-friendly, but also highly rugged and durable and able to last for at least ten years, despite rough handling and exposure to the harsh environmental conditions. For example, failure-prone electronically controlled motors and components could be replaced with an entirely mechanical positioning system, based on a structure of pulleys and counterweights that enables easy and 
accurate manipulation of the X-ray arm for all the required positions. The system could also integrate mechanical solutions to limit the range of motion, to avoid impact and shocks, instead of traditional electronically-based sensors. The ergonomic characteristics of this mechanical system must of course be suitable to the needs.

Finally, as water leakages may occur in small district hospitals located in tropical climates, the user interface could be based on a robust, tactile screen technology (such as the one used for automated teller machines), thus dispensing with fragile components like the keyboard and computer mouse.

iv) Lack of Skilled personnel. Even if a robust and well-adapted technology product were available, the lack of suitably qualified personnel can lead to improper use and product breakdown. Creative ways need to be proposed to facilitate the use of the technology even by people who lack complete and recent training. For example, the use of pictograms and images can improve ease of use, while at the same time removing language and cultural barriers. In the case of X-ray imaging, a user-friendly electronic guide could be integrated into the device, that may be regularly updated, e.g. through a standard GSM phone connection, in order to appropriately guide and upskill the operator according to their current level of expertise. Finally, the huge lack of trained radiologists in LMICs could seriously hinder the impact produced by deploying Xray systems. In addition to training doctors to interpret $X$-ray images, a solution with the capability to run computer-aided diagnosis (CAD), i.e. software tools based on Artificial Intelligence and deep learning techniques that offer guidance in the $X$-ray interpretation could be very valuable. It seems feasible to send X-ray images to remote sites via the GSM network, which could enable their interpretation by remotely located radiologists (tele-radiology).

v) Poor Governance. Major medical equipment needs to be correctly managed to guarantee continued effective results. In LMICs, issues with mismanagement can be frequent and affect all aspects, from the initial purchase of the technology, through to maintenance and repair, all the way to the final decommissioning and disposal. Especially problematic is the current practice in the industry of selling maintenance contracts, which have an annual fee. Often the funds lack at a later stage, and maintenance contracts are not renewed, which results in the maintenance being stopped. For large equipment, like imaging devices, this is especially problematic and represents a serious impediment to product sustainability. The problem requires a bold solution. One idea could be to do away with recurring maintenance contracts, but instead include several years of maintenance in the one-off initial purchase price (e.g. six years with an optional four-year renewal). Such an approach might help towards addressing the common challenge of poor financial planning in the management of major medical equipment. It is appears preferable to offer real additional digital services for a fee, than forcing the customer to "pay for uptime". This should be all the more feasible if the technology has been designed for robustness and durability. In addition to this, it is now also technically possible to monitor each machine remotely from a centralized location. This could be useful to predict and prevent potential breakdowns beforehand, via preventive maintenance planning, or to provide remote assistance.

All five factors discussed are interrelated and interdependent. Each one may significantly increase the risk of failure of a technology product. Of course, there is one more component, which is the cultural specificity of every region or country. Differences can be quite important, and these elements cannot be emphasized enough. As an example, the look and feel of a product is highly important and adequate attention devoted to this aspect can drastically improve the chances of product uptake, as in any market in the world.

In the case of diagnostic X-ray imaging, the user interface (touch screen) should be tested in several hospitals in LMICs and redesigned in an iterative manner, in order to ensure that the workflow and the graphics of the interface are appropriate for radiographers of diverse languages, cultures and skills. However, the overall system should also tested with radiographers in high-income countries, as it can be anticipated that it will also fill an unmet need in industrialized countries where healthcare costs are exploding. Being able to market the product in industrialized countries as well, not only represents an 
interesting potential market, but contributes also to brand credibility for customers in LMICs (who don't want a product "designed for the poor").

WHO? The Product Value Chain for a holistic perspective. LMIC markets are characterised by uncertainty, variations in available resources and major income disparities within the same country. Therefore, it is vital to gain as wide a perspective as possible about the targeted markets. In order to assure a systematic approach, we propose an expanded version of the value chain concept originally defined by Michael Porter ${ }^{21}$. According to Porter, the value chain refers to the organization of a company or a business unit as a whole; each group of activity in the company, such as operations, logistics or marketing, are a segment of the chain. Each segment consumes resources and adds value to the final output of the company. Our concept of value chain focuses on the product itself and not on the internal organization of a company. Thus, we refer to it as the Product Value Chain (PVC), where each segment of the chain is an activity that adds value to the final solution. A generic version of our proposed Product Value Chain tool is shown in Figure 3.

Analysing this value chain is vital as there is a tight relationship between each segment and the final product, as well as between the segments themselves. In order for the chain to be sustainable, each contributing segment needs to reliably provide value to the chain as well as retrieve value for itself, i.e. it must be suitably incentivised. For example, one segment is the transport of the product. The transporter brings value to the product by making it locally available, but also needs to earn a suitable margin for this task.

Each segment of the chain must be broken down to identify key suppliers, partners and other stakeholders who need to contribute to the sustainable availability and operation of the product. It also helps to identify potential challenges, which should be addressed as the technology is developed through the inputs of the key stakeholders. The information derived from the PVC analysis can thus be used to verify that all the specifications of the product are correct.

For the X-ray example, a simplified but specific Product Value Chain is shown in Figure 4. This exercise allows the identification of the key stakeholders who could be

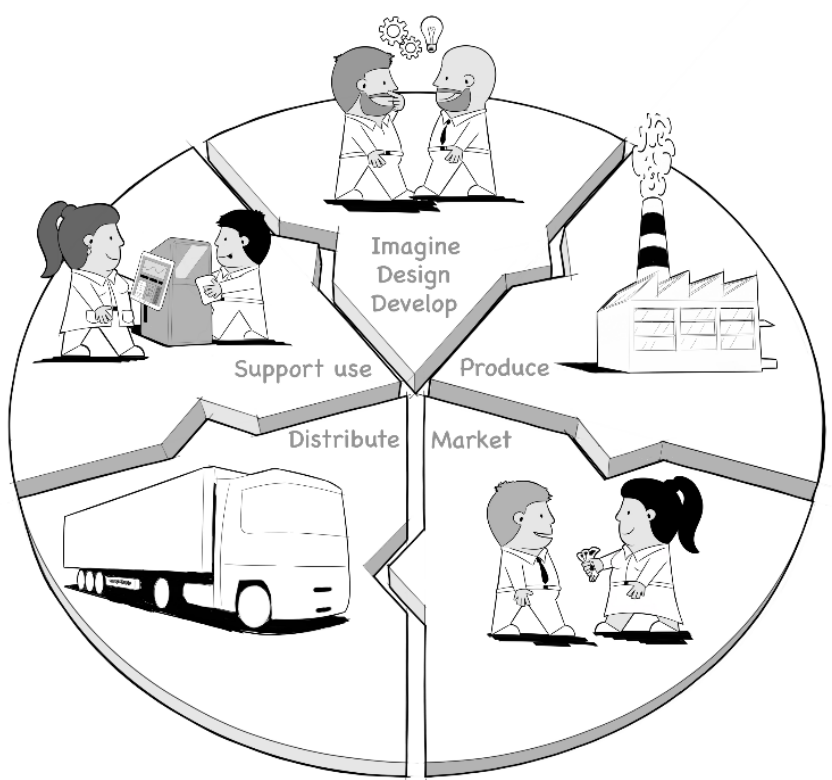

Figure 3: Generic Value Chain Canvas. This generic version should be expanded into a more detailed and technology-specific chain. the key enablers in the various chain segments. For Cameroon, conducting the analysis reveals that the National Radioprotection Agency is critical, because their approval is indispensable for the medical usage and proper commissioning of the technology. The same is true about the local radiographer organizations who are also vital players. Finally, this exercise also helps to bring out the importance of having continued and self-sustaining personnel training solutions, due to the high personnel turnover that is often found in LMICs. 


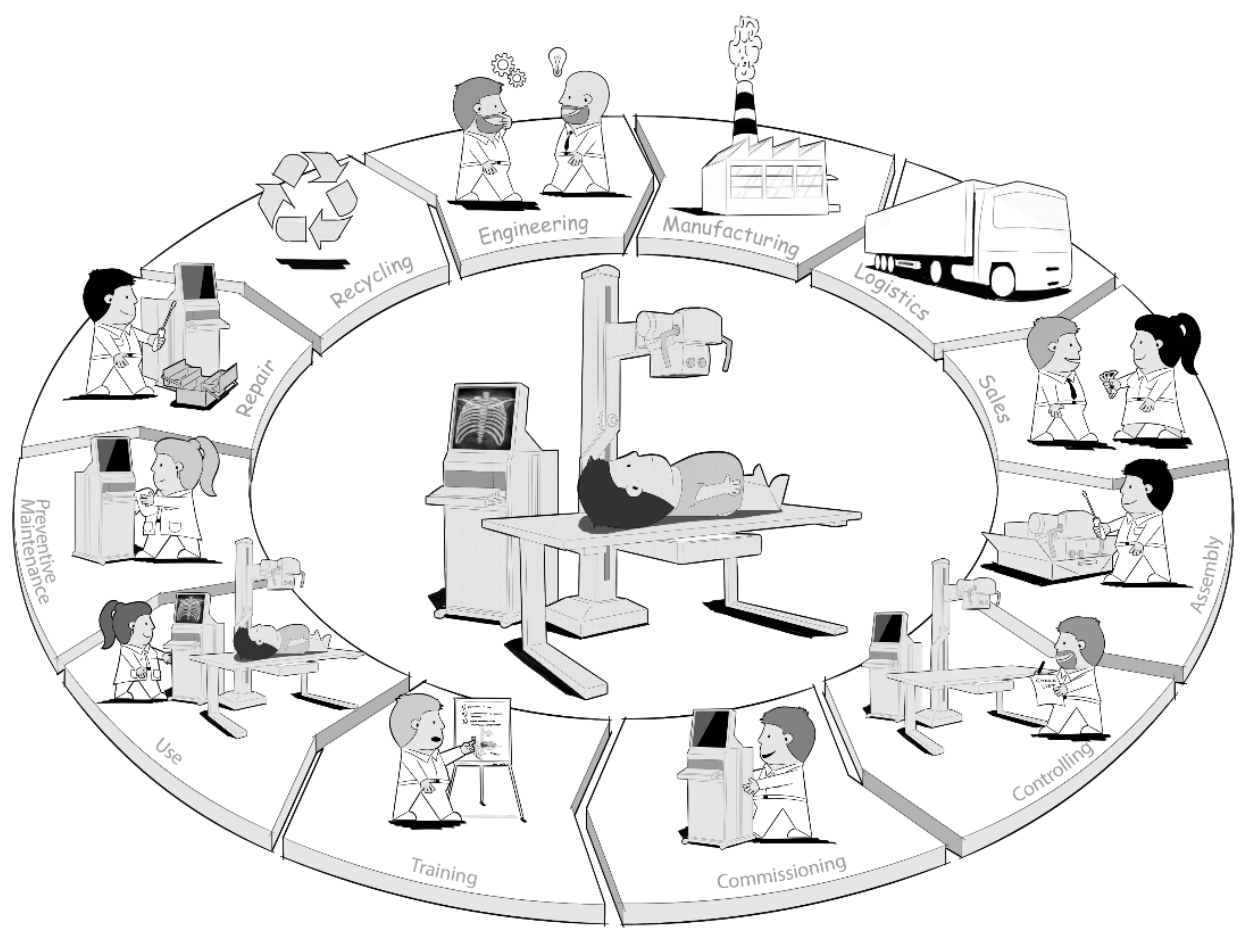

Figure 4 The specific value chain as defined in the case of the X-ray example

One of the important addroles of this tool is to help establish tradeoffs across the value chain. For example, developing an ultra-robust solution might reduce need for maintenance and repairs, but could increase the cost of the technology and make it harder to sell; or spending time to designing a simpler man-machine interface may reduce the need for training and so forth.

HOW? The Sustainable Business Model canvas. We also belong to the school of thought that believes that entrepreneurship is the best approach to deploy a technology-based product in sustainable manner at scale. One of the crucial components of entrepreneurship is the identification and elaboration of a suitable business for your product. Thus, it is important to understand revenue generation processes for any prospective product in LMIC markets, in order to ensure sustainable commercial viability. Most technology innovations require significant financial investments to convert them from a prototype and industrialise into a marketable product. Thus, a robust business model is also paramount for convincing and obtaining investors. We adopted the widely used business model canvas for traditional businesses originally described by Osterwalder and Pigneur ${ }^{22}$. This tool provides a systematic method to determine the mechanism(s) by which a new venture will generate income, as well as assist in developing the value proposition to contribute to sales and marketing activities. However, since we also aim to achieve social impact with the innovation, we modified this canvas to reflect impact and sustainability aspect over and above financial performance, giving rise to what we refer to as the Sustainable Business Model Canvas (Fig. 5). 


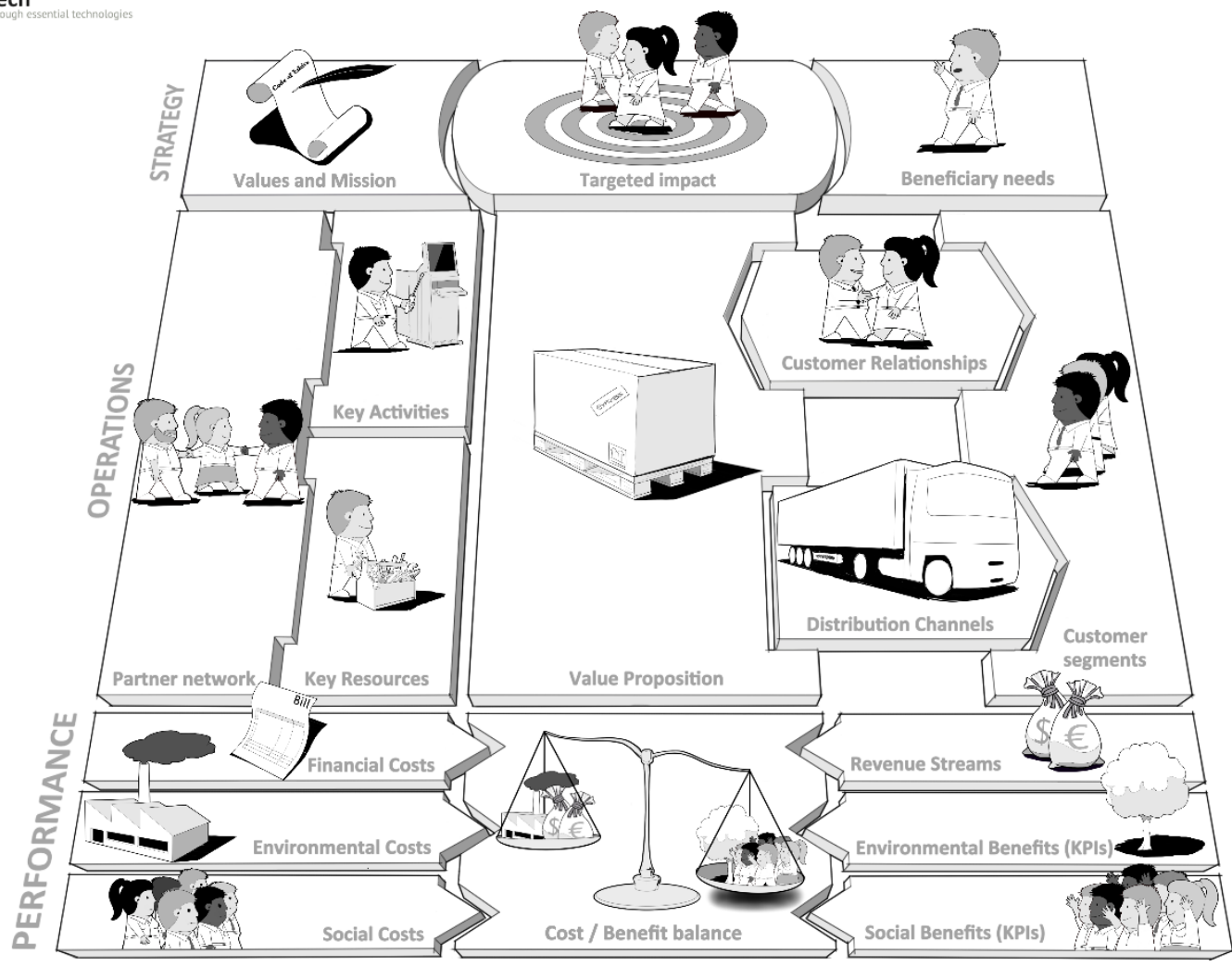

Figure 5 Sustainable Business Model Canvas. Modified from the Business model canvas by Osterwalder et al ${ }^{22}$. In the case of the $x$-Ray example, the content could be the following:

Values and Mission: The company will be making profit and it will be striving for sustainable global access to Xray imaging. It will strive to reach "zero downtime" in any contexts, and deploy at least $60 \%$ of its products in LMICs

Targeted Impact: Reduce mortality/morbidity due to trauma and pulmonary disease.

Beneficiary Needs: Access to affordable and good quality X-ray diagnosis

Customer Segments: Ministry of Health; private hospitals; international organizations; NGOs; development agencies.

Value Proposition: Reduced TCO; Robustness and durability; six-year warranty; digital and enabled for telemedicine and Computer Assisted Diagnosis.

Customer relationship: Local subsidiary and/or distributors (depending on customer segement).

Benefits: Financial - revenue from sale of machines and radiology services; Environmental - As the machine is digital, there is no chemical waste as in film-based machines; no lead; Social - contribution to universal health coverage by sustainable radiology access.

Key Activities: Engineering; Certification; Marketing; Shipping; Commissioning and Training; Maintenance. Key Resources: Engineers; Technicians; Radiology teachers; Sales force; Facilities; IP and Context knowledge. Partner Network: Contract Manufacturer; Freight Forwarder; Radiographers Associations; Medical School. Costs: Financial - Salaries, goods, ship, rent; Environmental - risk of no proper recycling of the battery; Social risk of X-ray overexposure, due to the ease of retaking a digital image.

We propose adding what we term the "Strategy" layer at the top of the canvas for representing the vision and strategy of the team. This will help the team to determine a common vision about issues such as their principle on profit: do they maximize profit or impact? If profit is made, will it be reinvested or will it be distributed to the shareholders and what proportion?

The Strategy layer also allows the definition of the impact we are seeking with the new innovation, as well as the identification of the potential beneficiaries. The beneficiary (e.g. patient) of a technology may not be the same as the customer (the one who pays for it) e.g. health ministry, private health care organization or 
health insurance. The proposed Sustainable Business Model Canvas makes this distinction clearly discernible.

The second "Operations" layer below is the traditional business model canvas 22 . This is where we define operational activities as well as customer strategy and segments. On the left hand side, the purpose is to define who will do what in generating the value proposition, which is the product itself along with other services sold to a specific customer segment. The right hand side defines the relations with the customer segments, i.e. how they are reached, and what kind of interaction is planned with them (aftersales services etc.). This is the revenue generating part.

Finally the "Performance" layer displays a triple bottom line involving financial, environmental and social dimensions. This layer prompts us to consider striking the right balance between economic viability and impact, between profitability and affordability, between potential social costs (i.e. collateral negative sideeffects of the technology on society) and benefits, between potentially generating environmental damage and improving life of the poorest. This exercise also forces us to reflect and clearly express our underlying drivers for the project. It is thus a very important and useful task towards creating clarity and alignment in the team concerning individual aspirations and personal philosophies.

With respect to our X-ray example, this tool allows us to address many questions relevant to the development of a complete business plan. One crucial question is who can and will pay for the acquisition of the technology? From the exercise of filling in the canvas five potential customer segments can be identified: i) Ministry of Health in the target countries; ii) Private hospitals (e.g. operated by faith-based organizations); iii) NGOs; iv) State development agencies (e.g. SDC in Switzerland, USAID in the USA, GIZ in Germany), v) international organizations such as UNICEF or the international Committee of the Red Cross (ICRC). The canvas also requires that we assign a specific value proposition for each of these "customer segments". In some cases, the product alone may be sufficient, whereas in others it might require the inclusion of ancillary services, such as on-site training of radiographers, maintenance by the company or even telemedicine to offset lack of requisite skills locally.

The use of this tool allows us to unveil and assess the global market potential of such a technology originally designed for LMICs. In the end it may turn out that a robust low-total-cost-of-ownership X-ray digital imaging solution may also be attractive even to the markets in high-income countries. It is well known that the challenges to contain and reduce healthcare is global concern.

For example, many physicians in high-income countries may wish to provide more services, such as radiology, to their patients but are unable to afford major equipment, as larger healthcare entities can. In principle, this market segment would also be able to accommodate a slightly higher price than in LMICs. This could permit a two-tier pricing structure and thereby increase the overall commercial attractiveness of the product and business plan for investors. Furthermore, such a dual market opportunity would also lend credibility to the product and potentially boost brand reputation in LMIC markets, since people in those regions are also sensitive to international brands.

\section{Discussion}

As mentioned before, the tools discussed above should be used in an iterative way. The process is summarized in figure 6. Most importantly, these tools should guide the development of the technology before it is transferred for implementation to the private sector. They are very powerful tools to de-risk the technology and try to maximize chances that the solution can be implemented and scaled. The whole process rests on three pillars: Cooperation, Interdisciplinarity and Entrepreneurship. 


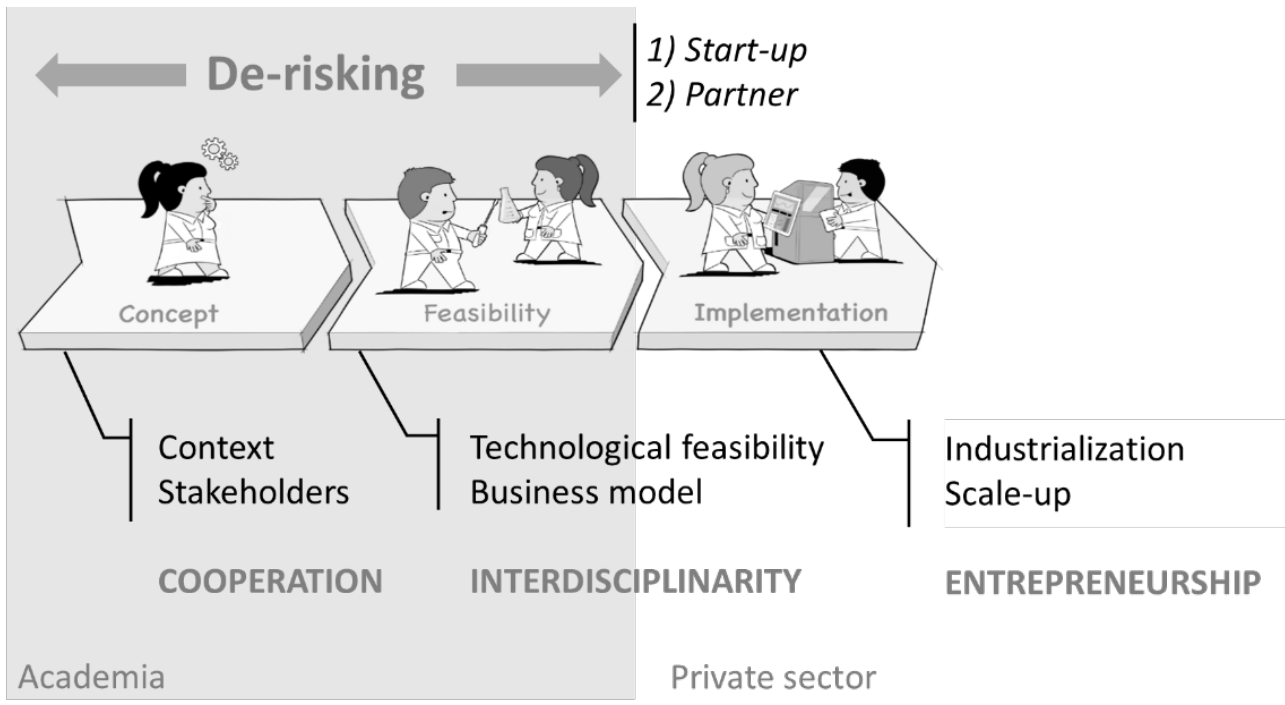

Figure 6 The overall process from concept to implementation, showing the three pillars of a successful approach: Cooperation, Interdisciplinarity and Entrepreneurship.

Cooperation: We cannot overemphasize the importance of a close collaboration with the local stakeholders in LMICs, which as expected, follows the well-known business mantra of "know your customer". However we also wish to stress the importance of international academic and research collaborations when developing solutions for LMICs. Consultations with key international organizations, such as the WHO and the ICRC, can offer invaluable inputs during the product development process. Altogether, these multistakeholder collaborations can provide a crucial contribution in understanding the needs, then defining and co-creating the solution.

Interdisciplinarity is key and becomes immediately evident when applying the tools: engineers or scientists alone cannot address the multiple facets of these complex challenges. The tools we propose can help "bring everyone to the same page" and identify creative solutions. Medical doctors, engineers, physicists, anthropologists, designers, logisticians and a wide variety of experts in other fields need to come together and co-develop solutions in order to maximize the chances of success.

Entrepreneurship is the cement that holds everything together. A sustainable impact can only be achieved if the project rests on a sound and economically viable value chain and business model. It is extremely important that researchers and academics recognise the need to be confronted with such aspects, which would help find solutions and trade-offs than can balance costs and stringent requirements. In our opinion, simply considering that this should be done by "the private sector...later" may be a missed opportunity to better tailor the innovation for its task and this may strongly undermine the chances of success for the technology. We believe that the tools discussed here may assist academics in adopting and assimilating entrepreneurial approaches in their innovation process, which may enable them to better understand the challenges in LMICS and be able to contribute effective solutions to them. For completeness, there are two topics worth mentioning that both tie up well with the discussion before and often a source of misconception when it comes to innovations for LMICs. The first issue concerns intellectual property: to patent or not to patent.

We believe that patents can be very useful, essentially for three reasons. Firstly, to protect the innovation against competition especially in the markets of industrialised countries. Secondly, since the industrialization of such an innovation requires appreciable private capital, a solid patent portfolio is helpful in negotiations with investors and contributes to the overall valuation of the enterprise. Thirdly, as academic inventors we may want to assure that the intended impact will be reached with our innovations, 
and patents can be an effective tools for policing this purpose. For example, a royalty-free sublicense could be given to a company along with a condition that it sells a certain pre-agreed proportion of their products in LMICs. This can potentially protect against "mission creep", e.g. when the licensee is confronted with lucrative opportunities that have higher margins in high-income markets, which could tempt them to focus their sales away from LMICs towards the more lucrative opportunities.

The second issue relates to regulatory aspects, as many LMICs do not have functional regulatory frameworks for medical products. Thus, it may be tempting to overlook norms and regulations, justifying it as a way to reduce costs and time-to-market for much needed products. Industrial norms and regulations define rules for safety and effectiveness and explain how medical devices should be developed and industrialized, as well as some aspects of how they are to be marketed. In our X-ray example, there are more than a dozen industry standards that apply and for HICs, e.g. EU or USA, and compliance to the Medical Devices Regulation (CE marking) or FDA requirements is mandatory. Compliance to those norms and regulations is essential, as for any products destined for high-income countries, regardless of the existence of local requirements in LMICs.

An x-ray solution has effectively been developed in line with the approach described in this article. Thanks to this methodology, we believe it may be on track towards solving the longstanding and reputedly difficult issue of global access to X-ray radiology, something that was highlighted by the WHO already in the $1970 \mathrm{~s}^{23}$. A start-up company has been formed which is currently industrializing the technology, with support from both European and African private investors. Of course it would be premature to claim success until the company has deployed a significant number of the devices in LMICs.

\section{Conclusions}

The primary mandate of academia and other public research centres is to extend the boundaries of knowledge and science, which of course is of crucial importance. On top of that, we argue that it is equally important to spare no efforts to make the benefits of these discoveries available to all humankind, including those at the bottom of the pyramid. We believe that more effort and priority is required to ensure that vital technologies are also accessible to those with limited means. For example, it is stunning that over 120 years after the discovery of the X-ray technology by Röntgen we still have two thirds of humanity with no access to this essential technology. In our view, academia can and needs to do more to promote scientific research and innovation that is destined at unmet needs in LMICs in essential technologies such as medical devices, or in the field of water and sanitation or nutrition. In entrepreneurship, unmet need equals opportunity. Tackling multi-dimensional problems involving a combination of stringent constrains related to costs, climate, infrastructure, usability etc., creates scientifically interesting challenges, which can lead to patentable innovations and novel interesting entrepreneurial opportunities. Finally, the technology example we discussed is complex, as X-ray devices involve hardware, software and mechanical components, as well as high voltage (up to $150^{\prime} 000$ Volts) and ionizing radiation technology. In addition, these devices are generally expensive and require complex and long sales cycles that involve several stakeholders, such as financial institutions, hospital management, radiologists, national radioprotection agencies, training institutions and commissioning sub-contractors. We thus hold a strong conviction that innovation for LMICs should not be confined solely to simple basic devices such as solar lanterns and cook stoves, but should also venture into more complex technologies if they are indeed needed and essential. We hope that the methodology we shared in this article can inspire researchers on how to approach technology innovation projects and improve their chances of success. We also hope that we have been able to transmit our views that we should embrace challenges that also involve complex technology solutions and not dwell exclusively on (over-) simplified solutions, sometimes referred to as "low-tech". Instead, we should strive to develop what could be termed "smart-tech", i.e. smartly conceived technologies designed to suit the most demanding and limited-resource conditions, but ultimately having a universal appeal and relevance. 


\section{AKNOWLEDGEMENTS}

Would like to express our special thanks to the University of Applied Sciences and Arts Western Switzerland (HES-SO), The Paul Scherrer Institute, The University Hospital of Canton de Vaud (CHUV), The Swiss Tropical and Public Health Institute (SwissTPH), the University Hospital Center and the CURES at the Ecole Nationale Supérieure Polytechnique in Yaoundé. The authors wish to thank Dr Gérard Escher, Fiona Fossati and Gene Saxon (EPFL), Steve Rudy (Gradian Health Systems), and Blaise Mentha (Privegest) for their help and advice with the manuscript.

\section{REFERENCES}

${ }^{1}$ World Health Organization (2010), Medical Devices: Managing the Mismatch. An Outcome of the Priority Medical Devices Project (World Health Organization, Geneva). ISBN 9789241564045.

${ }^{2}$ Bradley B, Chow S, Nyassi E, Cheng Y-L, Peel D, Howie S. The maintenance needs of oxygen concentrators in low-resource settings and implications for technician training: Experience from The Gambia. Health Tech. (2015).

${ }^{3}$ Peel D, Neighbour R, Eltringham RJ. Evaluation of oxygen concentrators for use in countries with limited resources. Anaesthesia. 2013;68(7):706-712.

${ }^{4}$ Gray AZ, Morpeth M, Duke T, et al. Improved oxygen systems in district hospitals in Lao PDR: a prospective field trial of the impact on outcomes for childhood pneumonia and equipment sustainability. BMJ Paediatr Open. 2017;1(1):e000083.

${ }^{5}$ Bakare AA, Graham H, Ayede Al, et al. Providing oxygen to children and newborns: a multi-faceted technical and clinical assessment of oxygen access and oxygen use in secondary-level hospitals in southwest Nigeria. Int Health. 2020;12(1):60-68.

${ }^{6}$ http://www.who.int/mediacentre/factsheets/fs363/en/

7 Darmstadt, Gary L et al. 2005. "Evidence-Based, Cost-Effective Interventions: How Many Newborn Babies Can We Save?" Lancet 365(9463): 977-88

${ }^{8}$ H. O. Amadi, O. A. Mokuolu, G. N. Adimora, S. D. Pam, U. S. Etawo, C. O. Ohadugha \& O. O. Adesiyun (2007) Digitally recycled incubators: better economic alternatives to modern systems in low-income countries, Annals of Tropical Paediatrics, 27:3, 207-214, DOI: 10.1179/146532807X220325

${ }^{9} \mathrm{~K}$. R. Olson and A. C. Caldwell, Designing an early stage prototype using readily available material for a neonatal incubator for poor settings 2010 Annual International Conference of the IEEE Engineering in Medicine and Biology, Buenos Aires, 2010, pp. 1100-1103.

${ }^{10}$ J. G. Meara, A. J. M. Leather, L. Hagander, B. C. Alkire, N. Alonso, E. A. Ameh, S. W. Bickler, L. Conteh, A. J. Dare, J. Davies, E. Dérivois Mérisier, S. El-Halabi, P. E. Farmer, A. Gawande, R. Gillies, S. L. M. Greenberg, C. E. Grimes, R. L. Gruen, E. A. Ismail, T. B. Kamara, C. Lavy, G. Lundeg, N. C. Mkandawire, N. P. Raykar, J. N. Riesel, E. Rodas, J. Rose, N. Roy, M. G. Shrime, R. Sullivan, S. Verguet, D. Watters, T. G. Weiser, I. H. Wilson, G. Yamey, W. Yip. Global Surgery 2030: evidence and solutions for achieving health, welfare, and economic development. The Lancet Vol 386 August 8, 2015

${ }^{11}$ J. A. Henry, E. Frenkel, E. Borgstein, N. Mkandawire, and C. Goddia, Surgical and anaesthetic capacity of hospitals in Malawi: key insights Health Policy and Planning 2015;30:985-994

${ }^{12}$ M. A. Rosen, J. B. Sampson, E. V. Jackson, R. Koka, A. M. Chima, O. U. Ogbuagu, M. K. Marx, M. Koroma and $B$. H. Lee: Failure mode and effects analysis of the universal anaesthesia machine in two tertiary care hospitals in Sierra Leone. British Journal of Anaesthesia 113 (3): 410-15 (2014)

${ }^{13}$ Radiology in Global Health: Strategies, Implementation and Applications. p.19. (2014) Eds. Mollura DJ, Lungren MP, (Springer-Verlag New York) ISBN 978-1-4614-0603-7

${ }^{14}$ Global Status Report on Road Safety (2015) World Health Organization. ISBN 9789241565066. 
${ }^{15}$ Niemeier D, Gombachika H, Richards-Kortum R (2014) How to transform the practice of engineering to meet global health needs. Science 345:1287-90. doi: 10.1126/science.1257085.

${ }^{16}$ Prahalad CK (2004) The fortune at the bottom of the pyramid. Wharton School Publishing (Philadelphia) ISBN 978-0-13-146750-7

${ }^{17}$ Richards-Kortum R, Oden M (2013) Engineering devices for low-resource health care. Science 342:10551057.

${ }^{18}$ Morra-Imas LG The road to results: designing and conducting effective development evaluations /ISBN 978-0-8213-7891-5 -- ISBN 978-0-8213-7911-0 (electronic).

${ }^{19}$ Sinha SR, Barry M (2011) Health Technologies and Innovation in the Global Health Arena. N Engl J Med 365:779-782, DOI: 10.1056/NEJMp1108040.

${ }^{20}$ Malkin RA (2007) Design of Health Care Technologies for the Developing World. Ann. Rev. of Biomed. Eng. 9:567- 587.

${ }^{21}$ Porter ME (1985). Competitive Advantage: Creating and Sustaining Superior Performance, (Simon and Schuster, New York).

${ }^{22}$ Osterwalder A, Pigneur Y (2010) Business Model Generation, (John Wiles \& Sons, New Jersey) ISBN 978 0470876411.

${ }^{23}$ Radiology and primary care. 1978. (Pan American Health Organisation Scientific Publication No 357.) Pan American Health Organisation, NW. Washington, DC 20037. 